Intervista a Luciano Cifaldi

\title{
Alessandro Meliciani
}

In un precedente incontro (Panni 2006) ci siamo soffermati sui vari modi con cui la documentazione autobiografica si presenta come patrimonio culturale, dalla corrispondenza privata ai diari alle interviste, alle carte trovate in un cassetto, alle memorie fra autofinzione e autobiografia, per soffermarsi alla fine su un particolarissimo 'diario illustrato' sulla vita di un lavoratore di Accadia, Vincenzo Palumbo, intitolato appunto Immagini di Accadia nei segni di Vincenzo Palumbo - figure di vita identiche nei paesi Dauni-Irpini e simili nei siti della spina dorsale dell'Appennino italico, Roma, Procaccini, 2000.

Nell'introduzione l'autore si presenta: «Affezionato al disegno ho cominciato a descrivere la vita che si viveva nel tempo passato, utilizzando anche i ricordi che mi raccontavano i miei nonni e i miei genitori. Così descrivevo 'come si viveva la miseria' con l'aiuto delle sole braccia. Allora non vi era limite alle ore di lavoro, perché non si aveva l'aiuto dei motori mentre le strade-mulattiere erano in cattivo stato».

Nei disegni di Vincenzo Palumbo, la vita di paese passa attraverso la descrizione di usi e costumi, gli interni delle case, dei negozi, dei luoghi di lavoro. Vengono minuziosamente descritte piccole e grandi cerimonie dall'uccisione del maiale al lavoro nei campi, alla trebbiatura, alla raccolta dell'uva, delle olive. Si vedono contadini con i loro attrezzi da lavoro, le massaie con gli oggetti della casa fino al calzolaio e ai vari artigiani con gli strumenti che garantiscono la riuscita delle loro attività. Si tratta di oggetti che gli anni del primo benessere faranno sparire dalle case dei contadini a cominciare dalle madie, dai tavoli di legno e marmo e tutti quegli utensili sostituiti da plastica e formica, primi segni dell'età del consumo. Gli stessi oggetti che Luciano Cifaldi comincerà a raccogliere e conservare.

Cominciamo però da più lontano, da quando Luciano partì dal suo paese. 
Nell'intervista Luciano ha parlato dei suoi inizi, delle origini del lavoro di restauratore. Ed è proprio in un cassetto di un mobile che ha trovato dei documenti ${ }^{1}$ dell'antico sindaco di Panni, Michele Rainone. Queste carte sebbene sparse, e senza un ordine cronologico, permettono di comprendere più di un secolo di storia di Panni. Luciano Cifaldi nel renderle note, ci ha consentito di ricostruire momenti della storia nazionale come il discorso del 6 marzo 1887 letto dal sindaco Rainone al popolo, dove si proclama: «da tre anni la nostra bandiera tricolore sventola sulle terre africane», alla cronaca locale come la nomina di Ufficiale alla Corona d'Italia del sindaco di Panni del 1907 con rispettiva festa, brindisi e finale sonetto dedicato al festeggiato. Storia e piccola cronaca contribuiscono a delineare il ritratto di un'epoca. I numerosi documenti inediti consentono una ricerca di verità e di vita nel tempo vissuto in un piccolo borgo d'Italia.

Luciano ha ricostruito l'intervista raccontandosi per iscritto. Ed ecco la narrazione della sua formazione professionale:

«Premetto che sono un autodidatta che non ha frequentato nessun corso di antiquariato, ma che tutto quello che ho imparato in questa professione lo devo esclusivamente ai miei MAESTRI D'ASCIA i cosidetti 'MAST' artigiani.

Adesso vi narro in sintesi la mia piccola grande storia.

Era la primavera dell' anno 1966, io poco più di 15 anni, apprendista falegname del mio paese d'origine (MONTAGUTO PROVINCIA D'AVELLINO), preso dalla voglia di far valigia ed approdare in una qualche parte d'Italia dove poter guadagnare qualche lira, decisi con l'accordo, anche se a malincuore, dei miei genitori di emigrare a Prato vicino Firenze.

Prato negli anni ' 60 ha ospitato per motivi di lavoro molti nuclei familiari del mio paese tra cui quello di una delle mie sorelle che aveva già formato una sua famiglia. Arrivai a Prato e trovai subito lavoro in un laboratorio artigianale di falegname. Nell'autunno del medesimo anno, e più precisamente il 4 novembre, dopo una settimana di piogge copiose che misero in ginocchio l'intera Toscana e buona parte del centro nord, l'Arno straripò, ruppe gli argini e si riversò sulla bellissima Firenze, distruggendo gran parte delle opere d'arte che si trovavano lungo la sua scia.

Io che abitavo ad un tiro di schioppo (come si suol dire) da Firenze, vuoi perché spinto dalla curiosità, vuoi perché giovane e curioso di vedere e di toccare con mano tutto quello che la catastrofica alluvione aveva causato, cinque o sei giorni dopo, mi recai a Firenze. Vidi così con i

\footnotetext{
1 A. Meliciani fa riferimento ad un insieme di documenti notarili e comunali, che tracciano un profilo di Panni fra fine Ottocento e primo Novecento che L.Cifaldi ha affidato all'Osservatorio Scientifico delle Memorie Scritte e Audiovisive.
} 
miei occhi le squadre ben organizzate di volontari, giunti da ogni parte d'Italia e oltre, salvare con un lavoro certosino oggetti di legno, stoffe, quadri, libri, e piccoli frammenti di qualsiasi cosa. Questi ultimi anche se a prima vista insignificanti risultarono preziosi e fondamentali per la ricostruzione degli oggetti suddetti. Lì capii il valore e l'importanza dell'antiquariato e dell'arte.

Da quell'avvenimento in poi nel mio tempo libero cominciai a restaurare mobili antichi, e, andando avanti con gli anni, questa mia passione si tramutò in professione.

Negli anni ottanta la mia avventura in Toscana finisce, perché nel frattempo sposatomi tornai al sud e più precisamente al paese nativo di mia moglie (Panni, Foggia), dove continuai a svolgere il mestiere di restauratore.

Fatalità volle che negli anni ottanta arrivò un catastrofico terremoto in Irpinia e nel subappennino dauno meridionale, che agevolò questo mio mestiere.

Le case danneggiate dal sisma dovevano essere risanate, e quindi molte persone si liberavano dei mobili, e, a volte, anche del loro contenuto (ecco il motivo del ritrovamento di quei documenti ${ }^{2}$ e altri oggetti in mio possesso), per poter permettere e facilitare l'opera dei muratori per la ricostruzione della case.

Ecco quello che ho capito dopo tanti anni di questa passione-lavoro:

OGNI APPROCCIO CON UN MOBILE O UN OGGETTO D'EPOCA È COME INTERPRETARE UN BRANO DI MUSICA CLASSICA.

IL RESTAURATORE DEVE INTUIRE, CAPIRE E SEGUIRE IL CAMMINO CREATIVO DELL 'ARTIGIANO ANTICO.

LA SODDISFAZIONE PER ME PIÙ GRANDE NON SONO I SOLDI, MA QUELLO CHE

DICONO GLI AMICI O CLIENTI SUGLI OGGETTI DA ME RESTAURATI, A CUI IO DO DI NUOVO LA VITA.

\footnotetext{
2 Vedi nota 4.
} 


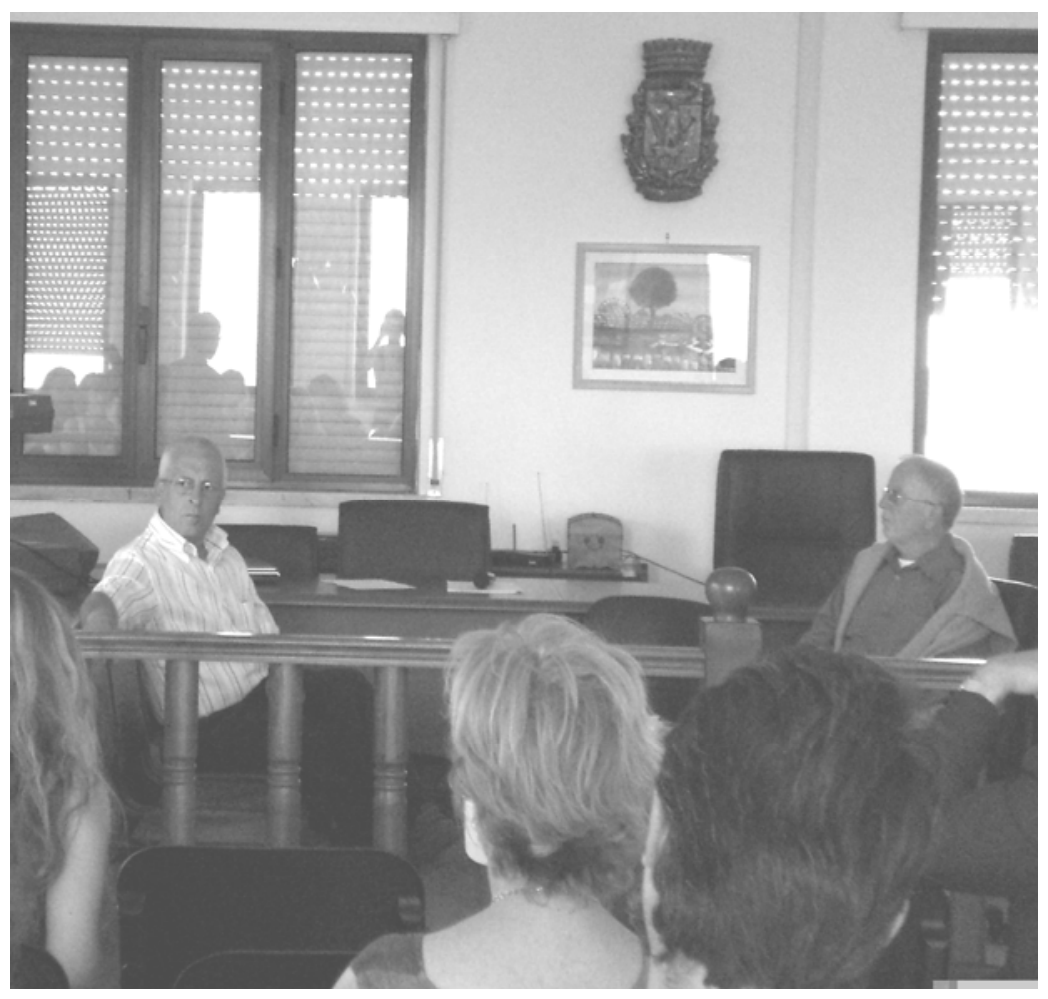

Luciano Cifaldi ed Alessandro Meliciani 